\title{
Indocyanine Green Fluorescence Measurement of Intestinal Transit and Gut Perfusion after Intestinal Manipulation
}

\author{
F.F. Behrendt ${ }^{a}, 1$ R.H. Tolba ${ }^{b} 1 \quad$ M. Overhaus ${ }^{a} \quad$ A. Hirner ${ }^{a} \quad$ T. Minor ${ }^{b}$ \\ J.C. Kalffa \\ aDepartment of Surgery and bSurgical Research Division, University of Bonn, Bonn, Germany
}

\section{Key Words}

Intestinal manipulation · Bowel perfusion · Intestinal transit $\cdot$ Indocyanine green-fluorescence measurement

\begin{abstract}
Background and Aims: Postoperative ileus is a common and poorly understood problem of abdominal surgery. The aim of this study was to measure postoperative intestinal transit and to evaluate bowel wall perfusion by a novel in vivo indocyanine green (ICG)-fluorescence measurement following intestinal manipulation (IM). Methods: Rats underwent a simple intestinal manipulation. Myeloperoxidase-positive cells in the muscularis were stained with the Hanker-Yates reaction and quantified histochemically. Bowel wall perfusion was determined directly and $24 \mathrm{~h}$ postoperatively using a laserfluorescence detection unit. Intestinal transit was visualized $24 \mathrm{~h}$ after IM. Results: IM resulted in a massive infiltration (155-fold) of neutrophils into the intestinal muscularis $24 \mathrm{~h}$ postoperatively. Bowel wall perfusion significantly decreased directly and $24 \mathrm{~h}$ following surgery ( 29 and $59 \%$, respectively). Gastrointestinal transit
\end{abstract}

Both authors contributed equally to this work.

Supported by BONFOR grants O-112.0006 and N-012.0029 and a grant from the Förderkreis Ferdinand-Sauerbruch to J.C.K. was similarly impaired and showed a reduction to $40 \%$ of the control values $24 \mathrm{~h}$ after IM. Conclusion: IM of the rat small intestine caused an impairment in bowel wall perfusion and microcirculation and a significant decrease in gastrointestinal transit. The ICG fluorescence measurement using the described system proved to be a simple and reliable method to evaluate intestinal transit and bowel wall microcirculation in vivo.

Copyright $@ 2004$ S. Karger AG, Basel

\section{Introduction}

Postoperative intestinal dysmotility or ileus often remains as an iatrogenic consequence following abdominal surgery [1]. This impairment of gastrointestinal motor function significantly affects the postoperative course of the individual patient and contributes to postsurgical morbidity and the length of hospital stay. In the United States the costs of postoperative ileus have been estimated to be approximately USD 1 billion/year [1].

Previously, we were able to demonstrate that surgical trauma to the gut results in a marked cellular infiltration into the bowel wall and especially into the smooth muscle layer [2]. In rodent models this inflammatory response was temporarily linked to smooth muscle dysfunction [3] and the causative relationship of leukocytic recruitment and inhibition of muscle function could be proven [4].

René H. Tolba, MD

Surgical Research Division, Department of Surgery

Rheinische Friedrich-Wilhelms-Universität Bonn

Sigmund-Freud-Strasse 25, DE-53105 Bonn (Germany)

Tel. +49 228287 6163, Fax +49228287 4263, E-Mail rtolba@uni-bonn.de 
Furthermore, we were able to demonstrate the identical mechanism in patients after abdominal surgery [5]. Besides the infiltrating leukocytes, the intestinal muscularis externa is hosted by a dense network of resident macrophages that are activated by surgical stress and other insults [2, 3, 6, 7]. Macrophages are known producers of a litany of inflammatory mediators [8]. Various kinetically active mediators (e.g. nitric oxide and prostaglandins) have been shown to be involved in postoperative muscle dysfunction $[9,10]$, but the initiators of this inflammatory process are unknown. Hypothetically, direct mechanical (surgical trauma), ischemic (local perfusion changes) and toxic (bacterial products) insults could play a major role in the beginning of this response. To find preventive mechanisms against this surgical conundrum, we need to study the initial events that lead to posttraumatic muscle inhibition. These events need to be investigated in animal models and also in the human situation. In the present study we therefore thought to evaluate a new noninvasive system that theoretically offers the possibility to study intestinal smooth muscle function by the quantification of gastrointestinal transit as well as intestinal perfusion in vivo. With the help of this IC-VIEW system we were able to measure intraluminal fluorescence in blood vessels and the bowel lumen after the administration of the non-toxic substance indocyanine green (ICG). The system proved capable of quantifying bowel wall (and mesenteric) blood perfusion and gastrointestinal transit in an in vivo setting in rats.

\section{Methods}

\section{Animals}

Male Sprague-Dawley rats (250-300 g body weight) were obtained from Harlan-Winkelmann (Borchen, Germany). All experiments were performed in accordance with the federal law regarding the protection of animals. The principles of laboratory animal care (NIH Publication No. 85-23, revised 1985) were followed. The animals were maintained on a 12-hour light/dark cycle and provided with commercially available chow (Altromin, Lage, Germany) and tap water ad libitum.

\section{Experimental Groups and Operative Procedures}

The small bowel of the animals was subjected to an easily standardized, mild intestinal manipulation as described previously [2]. In brief, animals were anesthetized with isoflurane inhalation and a midline abdominal incision was made. The small bowel was eventrated and laid onto moist gauze. Then the entire small bowel was lightly manipulated using moist cotton applicators. After manipulation, the laparotomy was closed by two layers of continuous sutures. All animals recovered rapidly from bowel manipulation without mortality. Bowel wall perfusion was determined directly after surgery and repeated $24 \mathrm{~h}$ postoperatively. Laparotomized, non-manipulated animals served as controls. For motility studies, gastrointestinal transit was evaluated $24 \mathrm{~h}$ after manipulation. Non-operated rats were used as controls. Specimens of these animals were used for histochemical studies.

\section{Histochemistry}

Histochemistry was performed on whole mounts of the distal jejunum as described before in detail [6]. Briefly, jejunal segments of $4 \mathrm{~cm}$ were cut from the bowel and immersed in iced Krebs-Ringer buffer (KRB) in a Sylgard ${ }^{\circledR}$-filled glass dish. The segments were pinned down along the mesenteric border; the bowel was opened, stretched and fixed in 100\% ethanol for $10 \mathrm{~min}$. Each segment was washed twice in $\mathrm{KRB}$, and mucosa and submucosa were stripped off under microscopic observation (MZ 8; Leica, Bensheim, Germany). The mucosa-free muscularis whole mounts were cut into $0.5 \times 1 \mathrm{~cm}$ pieces and used for staining procedures. Myeloperoxidase (MPO)positive cells were detected using a mixture of $10 \mathrm{mg}$ Hanker-Yates reagent (Sigma, Taufkirchen, Germany), $10 \mathrm{ml}$ KRB and $100 \mu \mathrm{l}$ hydrogen peroxide. The reaction was stopped with cold KRB. MPO+ cells were counted under a microscope (SM-LU, Leica) in 5 randomly chosen areas in each specimen at a magnification of $\times 160$.

\section{IC-VIEW System}

The noninvasive IC-VIEW system (Pulsion Medical Systems, Munich, Germany) contains an infrared source and a digital video camera with the special ability to detect near-infrared light in a special mode. Excitation light is provided by an IC-VIEW cameramounted near-infrared light source (NIR-light, laser class 3B, energy $0.16 \mathrm{~W}$, wavelength $780 \mathrm{~nm}$ ). ICG-derived fluorescence is detected by the digital IC-VIEW Video Camera and can be viewed in realtime. Results can be recorded on the camera tape and the digital data can then be transferred to a PC (Sony Vaio Laptop, PCG-3192; Sony Corp., Tokyo, Japan), stored and analyzed. Perfusion is evaluated automatically with the help of IC-CALC Software (IC-CALC Software; Pulsion Medical Systems, Munich, Germany) by definition of regions of interest and defining the start and endpoint of fluorescence rises.

\section{Measurement of Bowel Wall Perfusion}

Bowel wall perfusion was studied by an in vivo ICG-fluorescence measurement following intestinal manipulation $(0$ and $24 \mathrm{~h}$ postoperatively) and in controls ( $\mathrm{n}=5$ for each group). Animals were anesthetized with isoflurane inhalation and a midline abdominal laparotomy was made. The entire small bowel was eventrated onto moist gauze. Rats received $0.16 \mathrm{mg} / \mathrm{kg}$ of body weight ICG intravenously under illumination with a laser. The fluorescence signals of the blood perfusion were visualized by the IC-VIEW system. The perfusion was analyzed in three regions of interest by IC-CALC software. The reference region of the measurement was the superior mesenteric artery and the mean branches and was set at $100 \%$. For comparative measurements different areas of the mesentery and the small bowel wall were defined as the two other regions of interest. The software calculated the perfusion index in these regions as steepness of the curve of fluorescence intensity over time (pixel intensity per second). Results are given as a graphical plot and as the percentage of the perfusion index compared to the reference area.

\section{Functional Studies}

Gastrointestinal transit was measured in controls and manipulated animals $24 \mathrm{~h}$ postoperatively by visualizing the intestinal location of ICG ( $\mathrm{n}=5$ for each group). $22 \mathrm{~h}$ after bowel manipulation, 

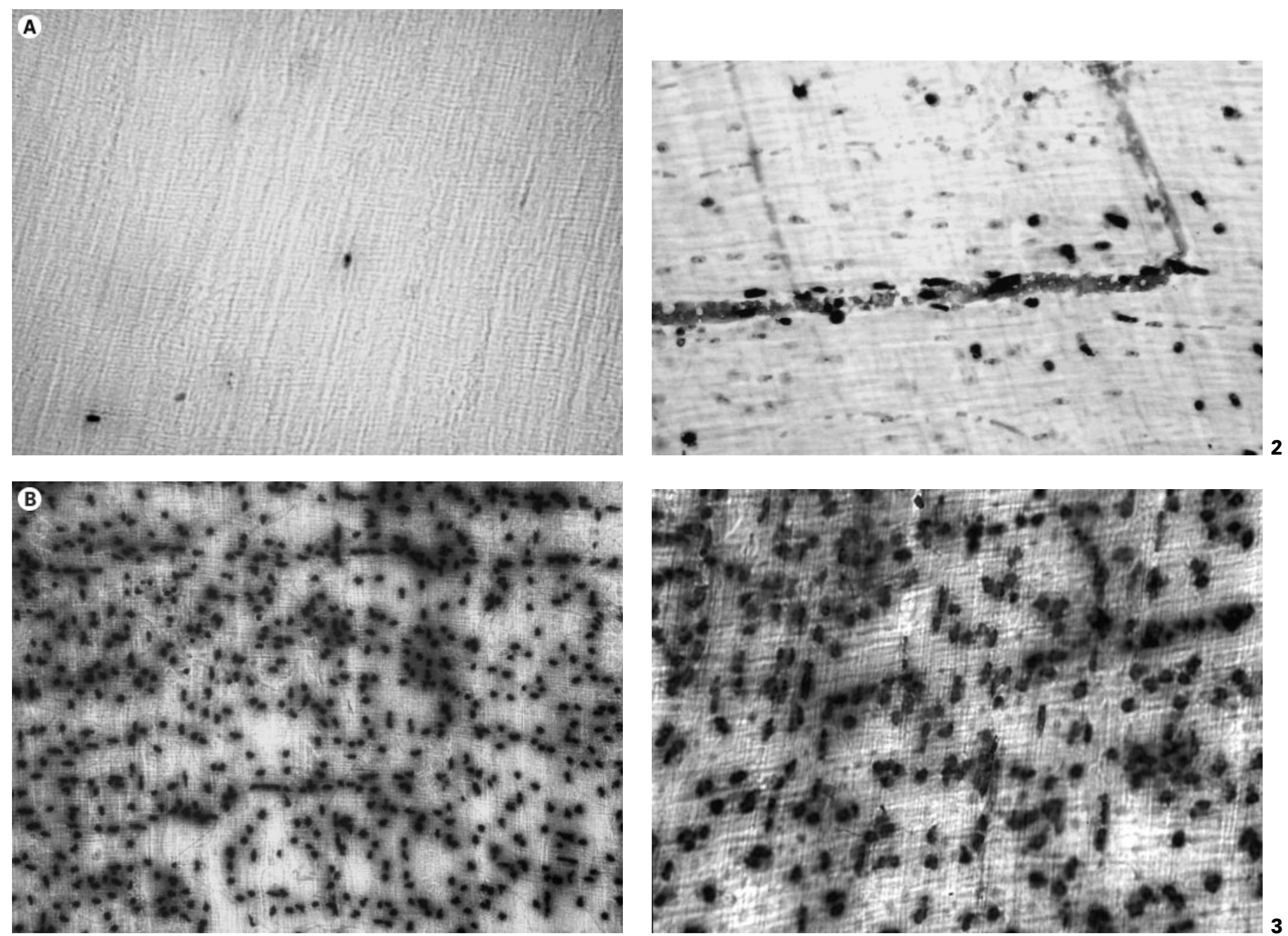

Fig. 1. Myeloperoxidase staining of rat muscularis whole mounts. A Whole mount from a control animal showing only a few MPOpositive cells (neutrophils) within the muscularis. B Intestinal manipulation caused a massive recruitment of MPO-positive cells $24 \mathrm{~h}$ postoperatively. Original magnification $\times 58$.

animals were lightly anesthetized and given $0.2 \mathrm{ml}(5 \mathrm{mg} / \mathrm{ml})$ ICG via a gastric tube into the stomach. Two hours after administration, in the control group and the manipulated group, ICG was visualized using the IC-VIEW System. Transit was evaluated in vivo and by ex vivo measurement of the bowel length that was passed by the marker.

\section{Drugs and Solutions}

A standard KRB was used with the following constituents (concentrations expressed as millimoles per liter): $\mathrm{Na}^{+}, 137.4 ; \mathrm{K}^{+}, 5.9$; $\mathrm{Ca}^{2+}, 2.5 ; \mathrm{Mg}^{2+}, 1.2 ; \mathrm{Cl}^{-}, 134 ; \mathrm{HCO}_{3}^{-}, 15.5 ; \mathrm{H}_{2} \mathrm{PO}_{4}^{-}, 1.2$, and glucose, 11.5. Sodium chloride and potassium chloride were obtained from Merck (Darmstadt, Germany). Sodium bicarbonate, sodium phosphate (monohydrate), glucose, calcium chloride dihydrate, magnesium chloride hexahydrate was purchased from Sigma (Taufkirchen, Germany).

Fig. 2. Myeloperoxidase histochemistry of a muscularis whole mount $6 \mathrm{~h}$ after intestinal manipulation. The muscularis is infiltrated with neutrophils and these cells are also visible inside smaller vessels. Original magnification $\times 51$.

Fig. 3. Myeloperoxidase (MPO) histochemistry of a muscularis whole mount $24 \mathrm{~h}$ after intestinal manipulation. The muscularis is densely infiltrated with neutrophils and these cells show degranulation and release of MPO granules. Original magnification $\times 58$.

ICG was purchased from Pulsion (ICG-PULSION; Pulsion Medical Systems, Munich, Germany).

\section{Data Analysis}

Data were compiled as mean \pm standard error of the mean.

Statistical analysis was performed using the unpaired Student's $t$ test.

Statistical significance was assumed at $p<0.05$. To analyze the bowel wall perfusion between the 3 groups (fig. 4; control, direct intestinal manipulation, $24 \mathrm{~h}$ after intestinal manipulation), one-way analysis of variance (ANOVA) was performed with the Dunnett's post hoc test. The control group was taken as reference. 


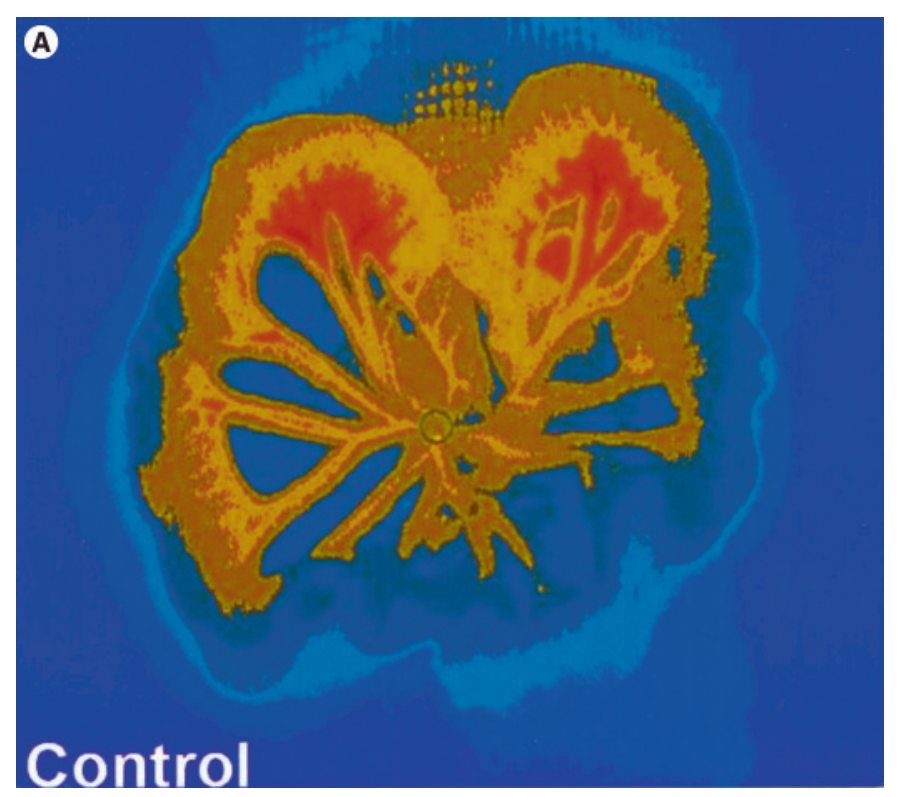

B

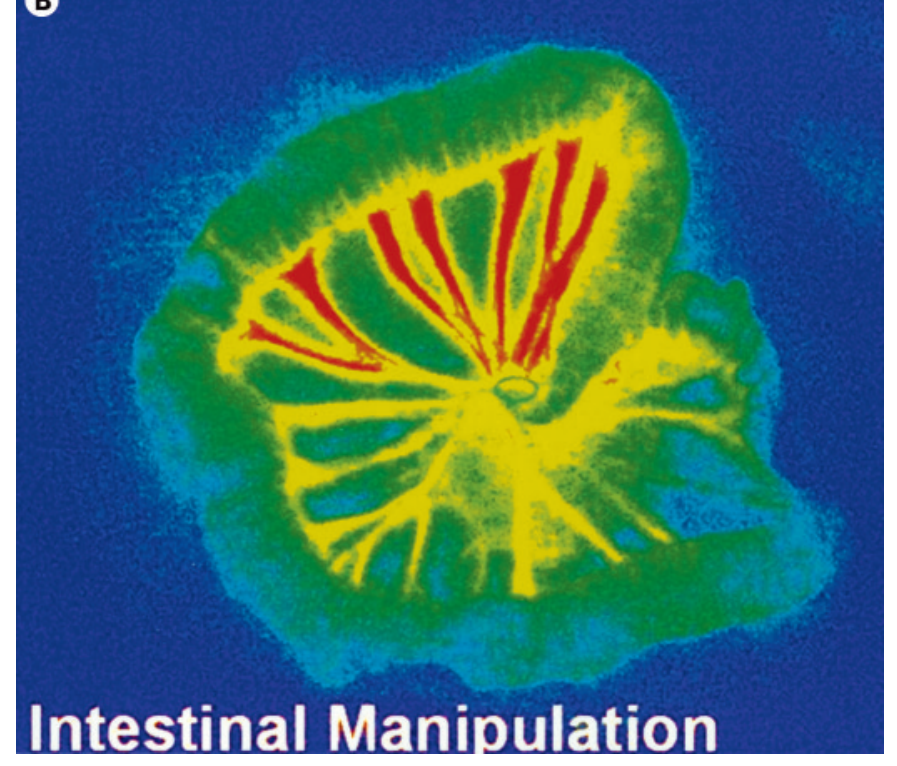

Fig. 4. ICG-fluorescence videography of the microcirculation in false colors. Pictures of the results of the ICG-fluorescence videography are given in false colors for better illustration. The colors stand for the fluorescence intensity. The perfusion in the reference area is shown in green and yellow. Blue indicates a lower perfusion than in the reference area and red a hyper-perfusion. Only the upper part of the picture was analyzed to avoid erroneous measurement due to less laser intensity in the lower part. A In control rats a homogenous perfusion with ICG was detectable. B Intestinal manipulation caused hyperemia in the mesentery and low perfusion in the bowel wall $24 \mathrm{~h}$ postoperatively.

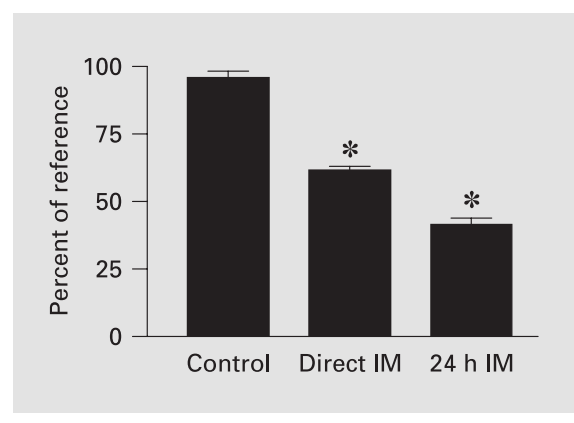

Fig. 5. Histogram of the small bowel wall perfusion in control and manipulated rats. In controls nearly no difference between the reference area and the bowel wall was measurable (95\%). Directly after intestinal manipulation the bowel wall perfusion decreased to $61 \%$ of the reference values and $24 \mathrm{~h}$ postoperatively the perfusion showed a reduction to $41 \%$. Control $=$ No intestinal manipulation; Direct IM = visualization of bowel wall perfusion directly after intestinal perfusion; $24 \mathrm{~h} \mathrm{IM}=$ visualization of bowel wall perfusion $24 \mathrm{~h}$ after intestinal perfusion. ${ }^{*} \mathrm{p}<0.05$ vs. control.

\section{Results}

\section{Muscularis Leukocytic Infiltrate}

Previously, we have shown that intestinal manipulation results in a significant infiltration of various leukocytes into the muscularis externa of the rat [2]. Therefore, we used the histochemical staining of neutrophils within the intestinal muscularis as a marker of the surgically induced local inflammation of the gut wall.

In muscularis specimens of non-manipulated control animals, MPO-positive cells were found only occasionally (fig. 1A). $24 \mathrm{~h}$ after intestinal manipulation, we were able to demonstrate the massive recruitment of neutrophils into the intestinal muscularis (fig. 1B). The number of polymorphonuclear leukocytes increased 155 -fold (591.0 \pm 39.9 vs. $3.8 \pm 0.9$ cells $/ \times 160$ magnification, $\mathrm{p}<0.05$ ) The neutrophils were found mainly outside of the vessels at $24 \mathrm{~h}$ (fig. 1B), but at earlier time points $(6 \mathrm{~h})$ we were able to see them attached inside the smaller vessels (fig. 2). Neutrophils showed degranulation and release of MPO granules in densely infiltrated areas (fig. 3).

\section{Perfusion of the Gut Wall}

We wanted to test if the IC-VIEW system is able to visualize and quantify perfusion changes within the bowel wall following surgical trauma.

Control animals (fig. 4A) showed a typical pattern of bowel wall perfusion. As shown in figure 4A, the marker showed a homogenous distribution throughout the entire 

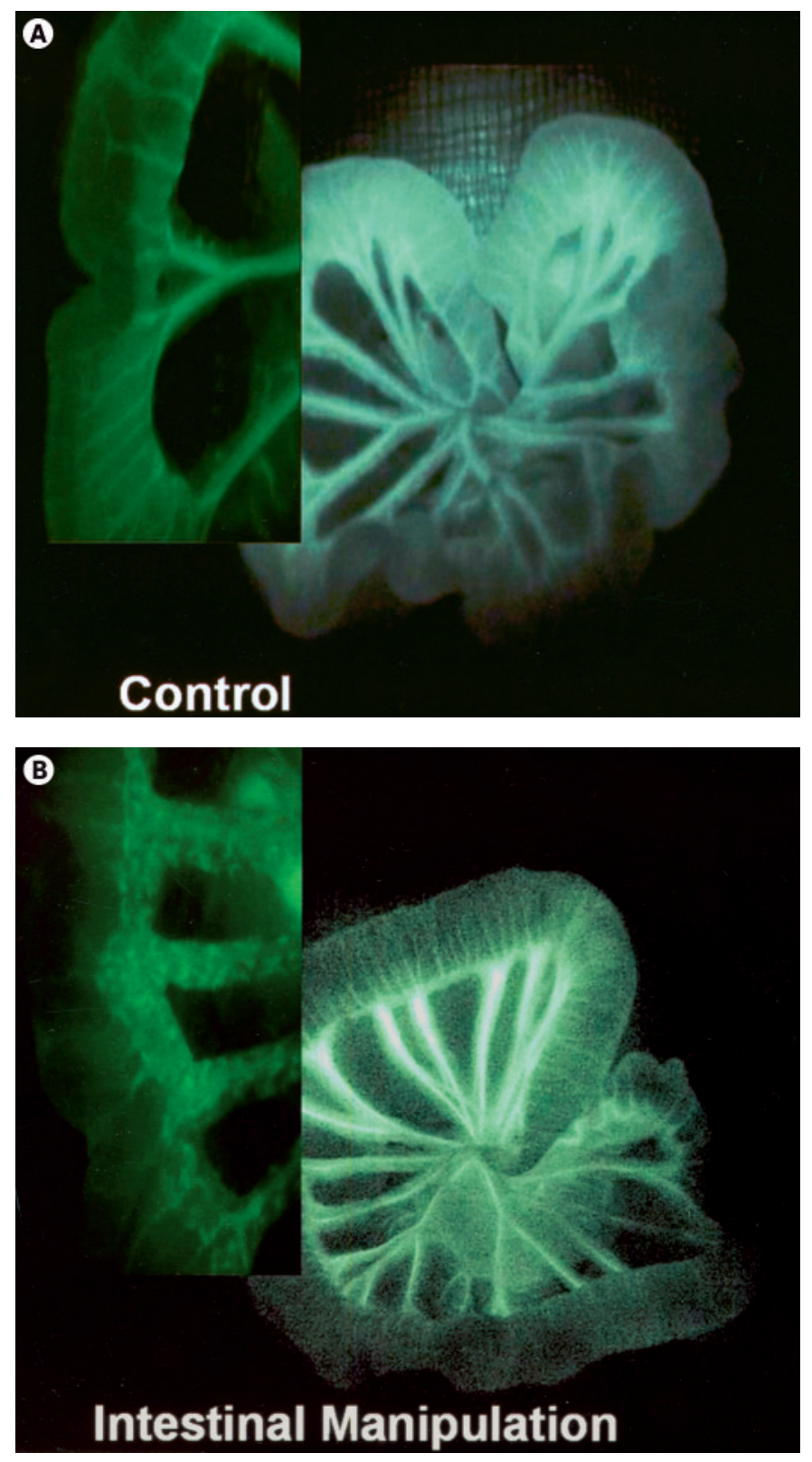

Fig. 6. A In control rats ICG was completely intravascular. B Intestinal manipulation caused an increase in the permeability. In the mesentery and the bowel wall we could detect ICG extravasation that is most likely extravasated leukocytes. Original magnification $\times 42$.

intestine. Early, within seconds after the intravenous application of ICG, the intensity of the signal was highest inside the mesenteric vessels. Within the bowel wall there was a notable decrease in signal from the mesenteric to the anti-mesenteric side of the bowel. The small arterial vessels on the lateral bowel wall are easily detectable in the figures $4 \mathrm{~A}$ and $6 \mathrm{~A}$.

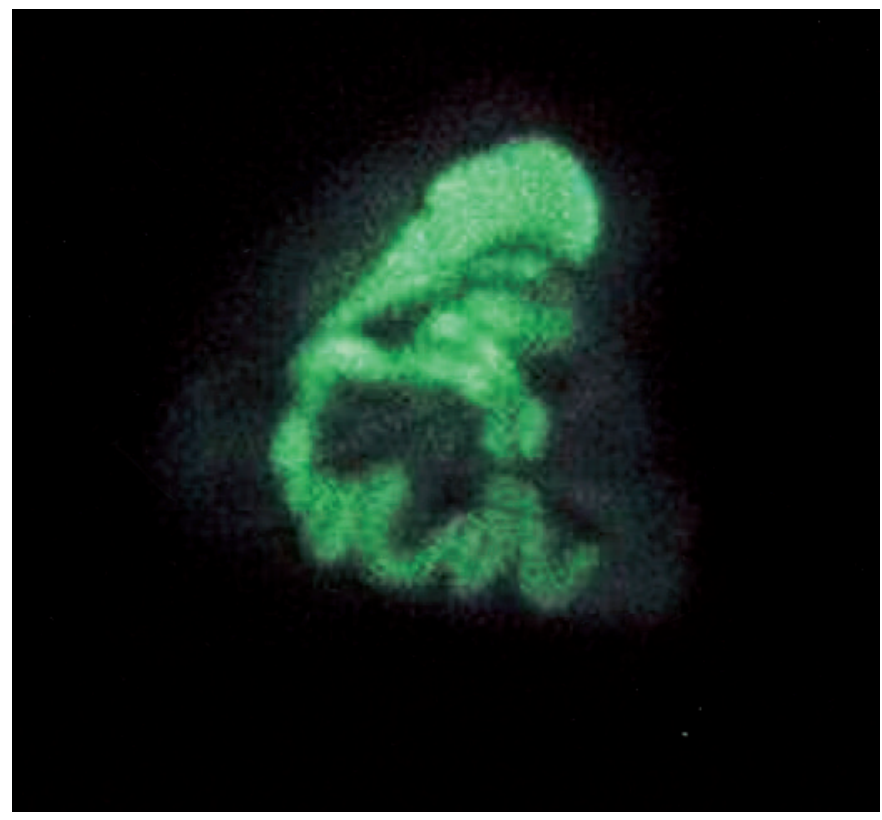

Fig. 7. Visualization of gastrointestinal transit in vivo using the ICVIEW system. A homogenous ICG signal from the stomach to the mid-small bowel is detectable.

All small bowel loops and the large bowel were perfused simultaneously with no local difference in marker distribution. Measurement of the perfusion showed nearly no difference between the bowel wall and the reference area (95\% perfusion index). Intestinal manipulation resulted $24 \mathrm{~h}$ postoperatively in even stronger signals in the mesentery (fig. 4B), most likely as a consequence of tissue edema, inflammation, extravasates and possible arteriovenous shunts. In the bowel wall signals were markedly weaker and showed a patchy and uneven distribution with areas of various perfusion states. Intestinal manipulation resulted in a $29 \%$ reduction in the perfusion index directly after the surgical procedure and, $24 \mathrm{~h}$ following manipulation, perfusion was decreased by $59 \%$ compared to the control group (fig. 5).

After intravenous application ICG rapidly and completely binds to plasma proteins. Extravasation of this marker is normally prevented if vascular permeability is not affected.

In control animals our findings were able to confirm this as shown in figure 6A: ICG remained completely intravascular. On the contrary, $24 \mathrm{~h}$ after intestinal manipulation, ICG extravasation outside the vessels was detected in the mesentery and also in the bowel wall (fig. 6B). There were no signs of hemorrhage into the 


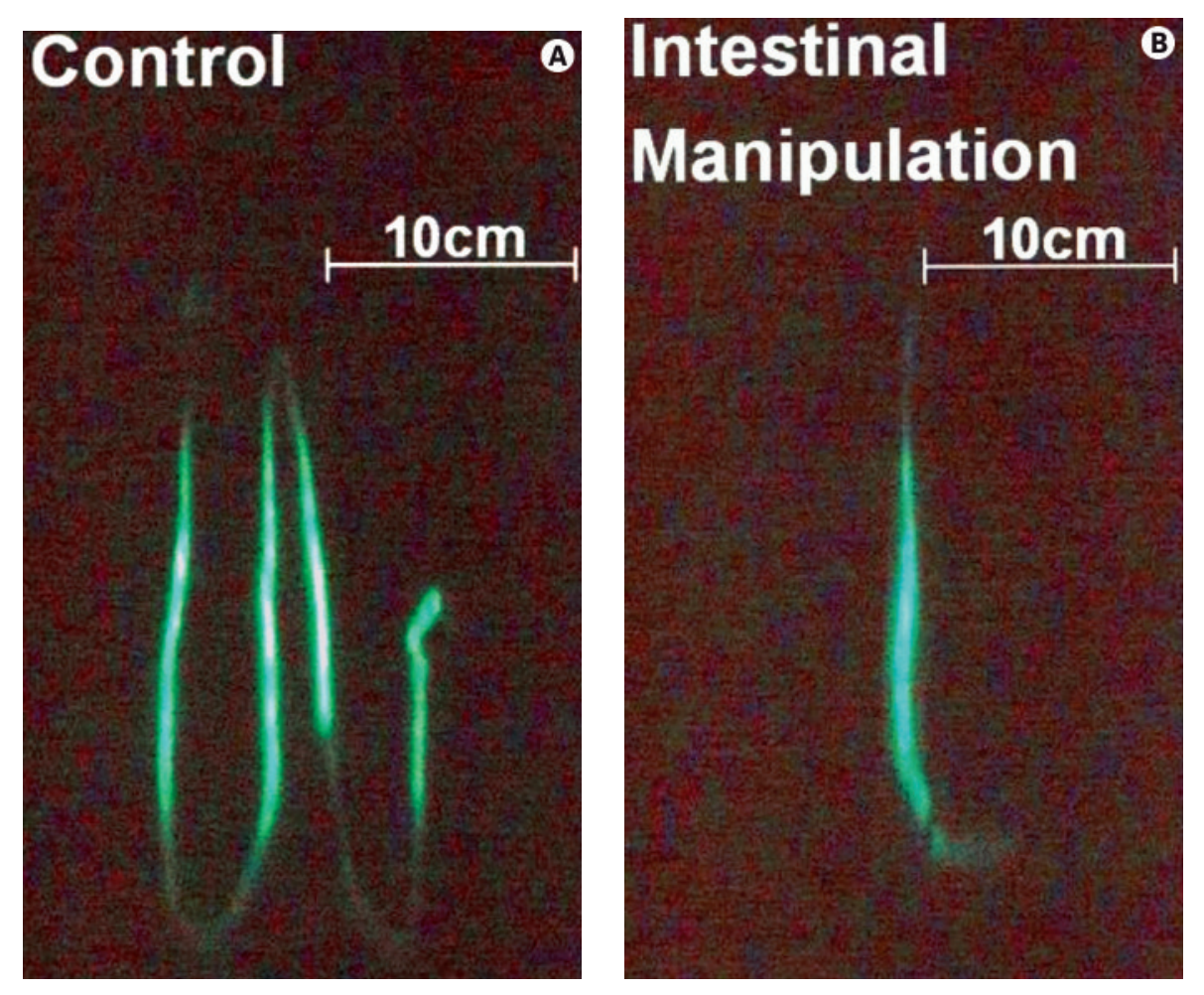

Fig. 8. Gastrointestinal transit from a control rat and a surgically manipulated animal. The ICG was administered orally and after laparotomy visualized with the IC-VIEW System. A In control animals a homogenous ICG signal could be detected in the whole small bowel reaching the cecum. B $24 \mathrm{~h}$ after intestinal manipulation ICG migrated only to the mid-jejunum. C Histogram of the quantified gastrointestinal transit. The intestinal manipulation resulted in a significant delay in rats $24 \mathrm{~h}$ postoperatively in contrast to the controls. ${ }^{*} \mathrm{p}<0.05$.

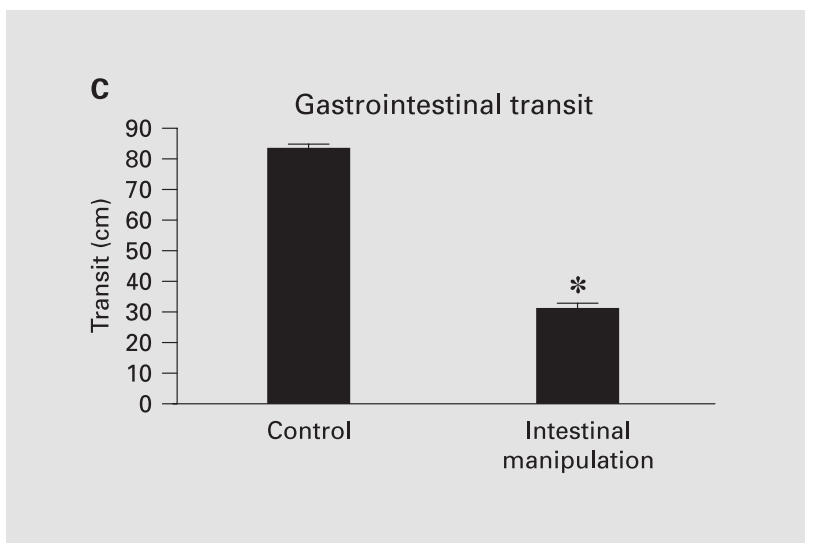

small bowel wall or hematomas in the mesentery. ICG can be used for vital staining of leukocytes [11], and therefore we hypothesize that these extravasates are most likely emigrated leukocytes, as we have seen in histochemical staining.

\section{Gastrointestinal Transit}

In previous studies of gastrointestinal transit, the gut had to be completely excised and studied in vitro after ingestion of a fluorescent marker. Therefore, with the help of the IC-VIEW system we wanted to prove if gastrointestinal transit can also be measured in vivo without the need of sacrificing the animal, leaving the bowel intact for possible further studies on these organs.

Gastrointestinal transit with this new system was studied in vivo as well as ex vivo. After laparotomy and eventration of the intestines, the intraluminal passage of ICG could easily be visualized with the IC-VIEW system (fig. 7). The difference in transit in control and manipulated animals was evident. In control animals a homogenous ICG signal was detected from the stomach to the end of the cecum. The distance of the ICG migration was 83 $\pm 1.57 \mathrm{~cm}$ (fig. $8 \mathrm{~A}, \mathrm{C}$ ). Intraluminal passage of the marker in animals that had undergone intestinal manipulation 
showed a decrease to $40 \%$ of control values $(31 \pm$ $1.73 \mathrm{~cm}$; fig. $8 \mathrm{~B}, \mathrm{C})$.

These data were confirmed by transit measurements of orally administered fluorescent dyes that were quantified in vitro by our previously reported technique [3] (data not shown).

\section{Discussion}

The development of postoperative ileus is a common feature of abdominal surgery. The underlying pathogenesis is poorly understood and most likely multifactorial [1, 12-14].

In previous studies we were able to show that intestinal manipulation of the rat small bowel caused massive inflammation within the muscularis that was subsequently followed by smooth muscle dysfunction leading to motility inhibition and ileus [4]. The histochemical and immunohistochemical stains of muscularis whole mounts have demonstrated a massive extravasation of leukocytes from within the vessels into the muscle layer [2]. The functional impairment of intestinal smooth muscle has been extensively studied in vitro by contractility measurements in various animal models $[2,10]$. Furthermore, gastrointestinal transit has been proven to be significantly slowed following surgical intestinal trauma in rodents [3]. Besides animal models we have demonstrated postoperative motility changes in the human situation following abdominal surgery and small bowel transplantation [5, 15]. To find preventive mechanisms against this surgical conundrum, we need to study the initial events that lead to posttraumatic muscle inhibition. In the present study we therefore sought to evaluate a new noninvasive system that theoretically offers the possibility of quantifying intestinal transit as well as intestinal perfusion in vivo. With the help of the IC-VIEW system we were able to measure the intraluminal fluorescence of blood vessels and the bowel lumen after the administration of the nontoxic substance ICG. Therefore, the system proved capable of quantifying gastrointestinal transit and bowel wall (and mesenteric) blood perfusion in an in vivo setting in a laparotomized rat.

In our previous experiments we noted that intestinal manipulation resulted in a marked instant hyperemia of the gut wall. Hyperemia is one of the principle symptoms of inflammation and is mediated by the release of local mediators like nitric oxide, prostaglandins, adenosine and others. Phagocytes are potent regulators of inflammation [8, 16-18]. Polymorphonuclear leukocytes are known to be one of the main leukocyte populations that infiltrate damaged tissues. Our data demonstrate that leukocyte recruitment leads to extravasation of the cells and subsequent liberation of intracellular granules, as shown by the degranulation of MPO granules in muscularis wholemount histochemistry. Substances liberated from these recruited phagocytes and also from the dense network of resident muscularis macrophages, like nitric oxide, prostaglandins, proteases and free radicals, lead to tissue damage and permeability changes in the microvessels [19-23]. The direct visualization of ICG within the vessels and tissues enabled in vivo evaluation of tissue perfusion and under $\times 60$ magnification also of the microcirculation of the bowel wall and mesentery. Despite the hyperemia in the mesentery, we found a marked reduction in the perfusion and microcirculation of the bowel wall directly following the surgical procedure, and this was even more pronounced $24 \mathrm{~h}$ postoperatively. These circulation changes will lead to an impaired nutritional supply to the bowel wall resulting in cell damage and increased inflammation. The perivascular ICG signal we detected is most likely a sign of endothelial cell damage and dysfunction with extravasated leukocytes, because there were no signs of hemorrhage or hematoma visible. We hypothesize that activation of resident macrophages within the muscularis externa leads to an upregulation of adhesion molecules and inflammatory mediators (e.g. IL$6, \mathrm{TNF}-\alpha$, etc.) and this leads to massive extravasation into the bowel wall as well the mesenteric tissue [4, 24]. ICG can be used for vital staining of leukocytes [11], and therefore we hypothesize that these extravasates are most likely emigrated leukocytes as has been seen in histochemical staining.

In our previous studies we were able to demonstrate that the amount of leukocyte infiltration into the smooth muscle layer varies with different injuries. The mechanical aspects of intestinal manipulation always produced severe cellular infiltration. The exact underlying mechanism is the subject of further investigations.

Measurement of the microcirculation of the intestine has been described before in other studies [25-28]. Most of the described systems have the major disadvantage that they are not easy to use in the human intraoperative situation. The IC-VIEW system is used in various conditions, intra- and postoperatively, to measure blood perfusion and microcirculation [29-32]. It has been proven effective and reliable in the settings of intraoperative assessment of vascular flow, evaluation of abdominal wall perfusion and intraoperative measurement of skin-flap viability $[33,34]$. Our study is the first to use the system for 
perfusion and transit measurements of the bowel in rodents.

Using this method we further investigated gastrointestinal transit in vivo for the first time, without any exposure to X-rays as in normal treatment with a barium meal. In other previous procedures, for example with Evans blue, transit could be investigated without X-ray, but the animals had to be sacrificed before measurement [35]. Thus, we were able to measure the consequences of intestinal manipulation and the resulting inflammation on the perfusion and transit of the small bowel in living animals. We have demonstrated a direct link between postoperative gut wall inflammation and inhibition of intestinal smooth muscle function [4]. In previous studies we have shown that muscle contractility of the small and large bowel is markedly decreased by surgical trauma [2, 36]. We found a significantly reduced gastrointestinal transit following intestinal manipulation as described in our previous in vitro experiments [2]. Intraluminal transit could be followed directly after the procedure or measured later in the postoperative period. In addition, the bowel segments could be used for other experimental methods (ex- traction of proteins, nucleic acids, etc.) from the same animals, because the intraluminal marker does not leave the lumen and did not interfere with following techniques. This method for transit studies proved to be superior to the technique we used previously [3]. It was less time-consuming, cheaper, easy to handle, highly reproducible and decreased the number of animals needed for the study, because it left the bowel intact.

In conclusion, this study shows that, by using the ICVIEW system, gastrointestinal transit and bowel wall perfusion can be quantified online in vivo. Besides the known postsurgical inflammatory response and subsequent dysmotility, we found marked hyperemia of the mesentery and perfusion changes with diminished bowel wall microcirculation in the small bowel of rats. Hence, the ICG fluorescence measurement using the system described proved to be a simple, reproducible and reliable method to evaluate intestinal transit and bowel wall microcirculation in vivo. Therefore, this system seems suitable for use in vivo in clinical settings and might open new insights into postoperative bowel perfusion and gastrointestinal transit in man.

\section{References}

$>1$ Livingston EH, Passaro EP Jr: Postoperative ileus. Dig Dis Sci 1990;35:121-132.

$>2$ Kalff JC, Schraut WH, Simmons RL, Bauer AJ: Surgical manipulation of the gut elicits an intestinal muscularis inflammatory response resulting in postsurgical ileus. Ann Surg 1998; 228:652-663.

$\checkmark 3$ Kalff JC, Buchholz BM, Eskandari MK, et al: Biphasic response to gut manipulation and temporal correlation of cellular infiltrates and muscle dysfunction in rat. Surgery 1999;126: 498-509.

$\checkmark 4$ Kalff JC, Carlos TM, Schraut WH, Billiar TR, Simmons RL, Bauer AJ: Surgically induced leukocytic infiltrates within the rat intestinal muscularis mediate postoperative ileus. Gastroenterology 1999;117:378-387.

$\checkmark 5$ Kalff JC, Turler A, Schwarz NT, et al: Intraabdominal activation of a local inflammatory response within the human muscularis externa during laparotomy. Ann Surg 2003;237:301315 .

6 Kalff JC, Schwarz NT, Walgenbach KJ, Schraut WH, Bauer AJ: Leukocytes of the intestinal muscularis: Their phenotype and isolation. J Leukoc Biol 1998;63:683-691.

7 Eskandari MK, Kalff JC, Billiar TR, Lee KKW, Bauer AJ: Lipopolysaccharide activates the muscularis macrophage network and suppresses circular smooth muscle activity. Am J Physiol 1997;273:G727-G734.
8 Nathan CF: Secretory products of macrophages. J Clin Invest 1987;79:319-326.

>9 Kalff JC, Schraut WH, Billiar TR, Simmons RL, Bauer AJ: Role of inducible nitric oxide synthase in postoperative intestinal smooth muscle dysfunction in rodents. Gastroenterology 2000;118:316-327.

10 Schwarz NT, Kalff JC, Turler A, et al: Prostanoid production via COX-2 as a causative mechanism of rodent postoperative ileus. Gastroenterology 2001;121:1354-1371.

11 Matsuda N, Ogura Y, Nishiwaki H, et al: Visualization of leukocyte dynamics in the choroid with indocyanine green. Invest Ophthalmol Vis Sci 1996;37:2228-2233.

12 Condon RE, Frantzides CT, Cowles VE, Mahoney JL, Schulte WJ, Sarna SK: Resolution of postoperative ileus in humans. Ann Surg 1986; 203:574-581.

13 Dubois A, Weise VK, Kopin IJ: Postoperative ileus in the rat: Physiopathology, etiology and treatment. Ann Surg 1973;178:781-786.

14 Smith J, Kelly KA, Weinshilboum RM: Pathophysiology of postoperative ileus. Arch Surg 1977;112:203-209.

15 Turler A, Kalff JC, Heeckt P, et al: Molecular and functional observations on the donor intestinal muscularis during human small bowel transplantation. Gastroenterology 2002;122: 1886-1897.
16 Stuehr DJ, Nathan CF: Nitric oxide. A macrophage product responsible for cytostasis and respiratory inhibition in tumor target cells. J Exp Med 1989;169:1543-1555.

17 Johnson KJ, Varani J, Smolen JE: Neutrophil activation and function in health and disease. Immunol Ser 1992;57:1-46.

18 Cicalese L, Rastellini C, Rao AS, Stanko RT: Pyruvate prevents mucosal reperfusion injury, oxygen free-radical production, and neutrophil infiltration after rat small bowel preservation and transplantation. Transplant Proc 1996;28: 2611.

19 Grisham MB, Hernandez LA, Granger DN: Xanthine oxidase and neutrophil infiltration in intestinal ischemia. Am J Physiol 1986;251: G567-G574.

20 Kurose I, Granger DN: Evidence implicating xanthine oxidase and neutrophils in reperfusion-induced microvascular dysfunction. Ann NY Acad Sci 1994;723:158-179.

21 Gulati OP, Malmsten C, Ponard G, Gulati N: The local edemogenic effects of leukotriene $\mathrm{C} 4$ and prostaglandin E2 in rats. Prostaglandins Leukot Med 1983;10:11-17.

22 Lozano Y, Taitz A, Petruzzelli GJ, Djordjevic A, Young MR: Prostaglandin E2-protein kinase A signaling and protein phosphatases-1 and $-2 \mathrm{~A}$ regulate human head and neck squamous cell carcinoma motility, adherence, and cytoskeletal organization. Prostaglandins 1996; 51:35-48. 
23 Payne DK, Fuseler JW, Owens MW: Modulation of endothelial cell permeability by lung carcinoma cells: A potential mechanism of malignant pleural effusion formation. Inflammation 1994; 18:407-417.

24 Turler A, Schwarz NT, Turler E, Kalff JC, Bauer AJ: MCP-1 causes leukocyte recruitment and subsequently endotoxemic ileus in rat. Am J Physiol Gastrointest Liver Physiol 2002;282 G145-G155.

-25 Boros M, Massberg S, Baranyi L, Okada H, Messmer K: Endothelin 1 induces leukocyte adhesion in submucosal venules of the rat small intestine. Gastroenterology 1998;114:103114.

26 Gonzalez AP, Sepulveda S, Massberg S, Baumeister R, Menger MD: In vivo fluorescence microscopy for the assessment of microvascular reperfusion injury in small bowel transplants in rats. Transplantation 1994;58:403408.

27 Massberg S, Eisenmenger S, Enders G, Krombach F, Messmer K: Quantitative analysis of small intestinal microcirculation in the mouse. Res Exp Med (Berl) 1998;198:23-35.
28 Massberg S, Gonzalez AP, Leiderer R, Menger MD, Messmer K: In vivo assessment of the influence of cold preservation time on microvascular reperfusion injury after experimental small bowel transplantation. Br J Surg 1998;85: 127-133.

29 Eren S, Krein R, Hafemann B: Objective evaluation of the microcirculation in the skin with indocyanine green angiography (ICGA). A method for the clinic? Handchir Mikrochir Plast Chir 1995;27:307-314.

30 Green HA, Bua D, Anderson RR, Nishioka NS: Burn depth estimation using indocyanine green fluorescence. Arch Dermatol 1992;128: 43-49.

31 Rubben A, Eren S, Krein R, Younossi H, Bohler U, Wienert V: Infrared videoangiofluorography of the skin with indocyanine green Rat random cutaneous flap model and results in man. Microvasc Res 1994;47:240-251.
32 Still J, Law E, Dawson J, Bracci S, Island T, Holtz J: Evaluation of the circulation of reconstructive flaps using laser-induced fluorescence of indocyanine green. Ann Plast Surg 1999;42: 266-274.

33 Hoer J, Tons C, Schachtrupp A, et al: Quantitative evaluation of abdominal wall perfusion after different types of laparotomy closure using laser-fluorescence videography. Hernia 2002;6:11-16.

34 Raabe A, Beck J, Gerlach R, Zimmermann M, Seifert V: Near-infrared indocyanine green video angiography: A new method for intraoperative assessment of vascular flow. Neurosurgery 2003;52:132-139.

35 De Winter BY, Boeckxstaens GE, De Man JG, et al: Effect of different prokinetic agents and a novel enterokinetic agent on postoperative ileus in rats. Gut 1999;45:713-718.

36 Turler A, Moore BA, Pezzone MA, Overhaus M, Kalff JC, Bauer AJ: Colonic postoperative inflammatory ileus in the rat. Ann Surg 2002; 236:56-66. 